\title{
Molecular typing of lung adenocarcinoma with computed tomography and CT image-based radiomics: a narrative review of research progress and prospects
}

\author{
Jing-Wen $\mathrm{Ma}^{\wedge}$, Meng Li \\ Department of Diagnostic Radiology, National Cancer Center/National Clinical Research Center for Cancer/Cancer Hospital, Chinese Academy of \\ Medical Sciences and Peking Union Medical College, Beijing, China \\ Contributions: (I) Conception and design: M Li; (II) Administrative support: M Li; (III) Provision of study materials or patients: None; (IV) Collection \\ and assembly of data: None; (V) Data analysis and interpretation: None; (VI) Manuscript writing: Both authors; (VII) Final approval of manuscript: \\ Both authors. \\ Correspondence to: Meng Li, MD. Department of Diagnostic Radiology, National Cancer Center/National Clinical Research Center for Cancer/ \\ Cancer Hospital, Chinese Academy of Medical Sciences and Peking Union Medical College, Beijing 100021, China. Email: lmcams@163.com.
}

\begin{abstract}
Objective: The purpose of this paper was to perform a narrative review of current research evidence on conventional computed tomography (CT) imaging features and CT image-based radiomic features for predicting gene mutations in lung adenocarcinoma and discuss how to translate the research findings to guide future practice.
\end{abstract}

Background: Lung cancer, especially lung adenocarcinoma, is the leading cause of cancer-related deaths. With advances in the diagnosis and treatment of lung adenocarcinoma with the emergence of molecular testing, the prediction of oncogenes and even drug resistance gene mutations have become key to individualized and precise clinical treatment in order to prolong survival and improve quality of life. The progress of imageological examination includes the development of CT and radiomics are promising quantitative methods for predicting different gene mutations in lung adenocarcinoma, especially common mutations, such as epidermal growth factor receptor (EGFR) mutation, anaplastic lymphoma kinase (ALK) mutation and Kirsten rat sarcoma viral oncogene (KRAS) mutation.

Methods: The PubMed electronic database was searched along with a set of terms specific to lung adenocarcinoma, radiomics (including texture analysis), CT, computed tomography, EGFR, ALK, KRAS, rearranging transfection (RET) rearrangement and c-ros oncogene 1 (ROS-1), v-raf murine sarcoma viral oncogene homolog B1 (BRAF), and human epidermal growth factor receptor 2 (HER2) mutations et al. This review has been reported in compliance with the Narrative Review checklist guidelines. From each full-text article, information was extracted regarding a set of terms above.

Conclusions: Research on the application of conventional CT features and CT image-based radiomic features for predicting the gene mutation status of lung adenocarcinoma is still in a preliminary stage. Noninvasively determination of mutation status in lung adenocarcinoma before targeted therapy with conventional CT features and CT image-based radiomic features remains both hopes and challenges. Before radiomics could be applied in clinical practice, more work needs to be done.

Keywords: Lung adenocarcinoma; epidermal growth factor receptor (EGFR); Kirsten rat sarcoma viral oncogene (KRAS); anaplastic lymphoma kinase (ALK); conventional CT features; CT image-based radiomics

Submitted Jun 16, 2021. Accepted for publication Sep 03, 2021.

doi: $10.21037 /$ tcr-21-1037

View this article at: https://dx.doi.org/10.21037/tcr-21-1037

\footnotetext{
^ ORCID: 0000-0002-0966-9179.
} 


\section{Introduction}

Lung cancer remains the leading cause of cancer-related mortality worldwide $(1,2)$. Many studies have shown that the incidence rate of lung cancer in non-smoking women and the detection rate of lung cancer, especially lung adenocarcinoma, after the implementation of lung cancer screening program are both increasing year by year, and lung adenocarcinoma has become the most common subtype of lung cancer and has the highest gene mutation rate (3-8). In recent decades, the discovery of cancer driver genes and their functions in predicting targeted therapy effects have led to great success in developing methods for the diagnosis and treatment of advanced lung adenocarcinoma. Alterations in driver genes are closely related to the prognosis of patients (9-18), and the accurate identification of oncogenic gene mutations is essential for patients with lung adenocarcinoma. Therefore, research on the molecular mechanism of lung adenocarcinoma at the micro level is further increased. The driver genes of lung adenocarcinoma mainly include epidermal growth factor receptor (EGFR), anaplastic lymphoma kinase (ALK), and Kirsten rat sarcoma viral oncogene (KRAS). Rare gene alterations include rearranging transfection (RET) rearrangement and c-ros oncogene 1 (ROS-1), v-raf murine sarcoma viral oncogene homolog B1 (BRAF), and human epidermal growth factor receptor 2 (HER2) mutations. To detect these mutations, pathological biopsy is still the "gold standard". Of note, biopsy cannot assess all cancer lesions; repetitive biopsies increase the risk of local metastasis of tumours $(19,20)$, the economic burden of patients and the tolerance of patients. In view of these limitations, there is an urgent need for a noninvasive, repeatable, and costeffective technique for detecting driver gene mutations in lung adenocarcinoma.

Imaging examination, especially CT, is the most important noninvasive examination of lung cancer. There are many imaging features used to describe the conventional CT findings of lung adenocarcinoma. Ground glass opacity (GGO) is used to depict lung lesions that appear as hazy regions of increased opacity in the lung with preservation of bronchial and vascular margins, which is an important feature of lung adenocarcinoma $(21,22)$. There are still some other features, such as bubble-like lucency $(23,24)$, pleural retraction (25), and air bronchogram (22), which are also the common imaging descriptions for lung adenocarcinoma. However, conventional imaging features are subjective and lack quantitative objective basis, and some features overlap and lack specificity.

Radiomics, as a new detection technique used to predict gene mutations, combines conventional imaging technology with an advanced computer algorithm and changes the conventional reading method that extracts imaging information in a subjective and semiquantitative way, directly translating visual image information into quantitative characteristics (26). Radiomics has revealed that the macroscopic imaging characteristics of tumours are closely related to microscopic genetic changes $(27,28)$, which provides opportunities for molecular gene prediction in lung adenocarcinoma. The process of radiomics for molecular typing of lung adenocarcinomas includes six steps: (I) acquiring the images, (II) identifying the volumes of interest, (III) segmenting the volumes (i.e., delineating the borders of the volume with computer-assisted contouring), (IV) extracting and qualifying descriptive features from the volume, (V) using these to populate a searchable database, and (VI) mining these data to develop classifier models to predict outcomes either alone or in combination with additional information, such as clinical and conventional CT features (Figure 1). Radiomic features are divided into two categories: semantic features and agnostic features (29). Semantic features are those commonly used by radiologists to describe lesions, such as size, shape, location, and necrosis, while agnostic features are those that attempt to capture lesion heterogeneity through quantitative descriptors, such as histogram (skewness, kurtosis), Haralick textures, laws textures, wavelets, Laplacian transforms, Minkowski functionals, and fractal dimensions (29) (Table 1).

In recent years, there are many studies on predicting gene mutation phenotype of lung adenocarcinoma with conventional CT imaging features and CT image-based radiomic features. Therefore, in this article, we review the current research evidence on conventional CT imaging features and CT image-based radiomic features for predicting gene mutations in lung adenocarcinoma and discuss how to translate the research evidence to guide future practice. We present the following article in accordance with the Narrative Review reporting checklist (available at https://dx.doi.org/10.21037/tcr-21-1037).

\section{Conventional CT imaging features and CT image-based radiomic features predict EGFR, its subtypes, and drug resistance gene mutations in lung adenocarcinoma}

As the most common and most concerning oncogenic gene 


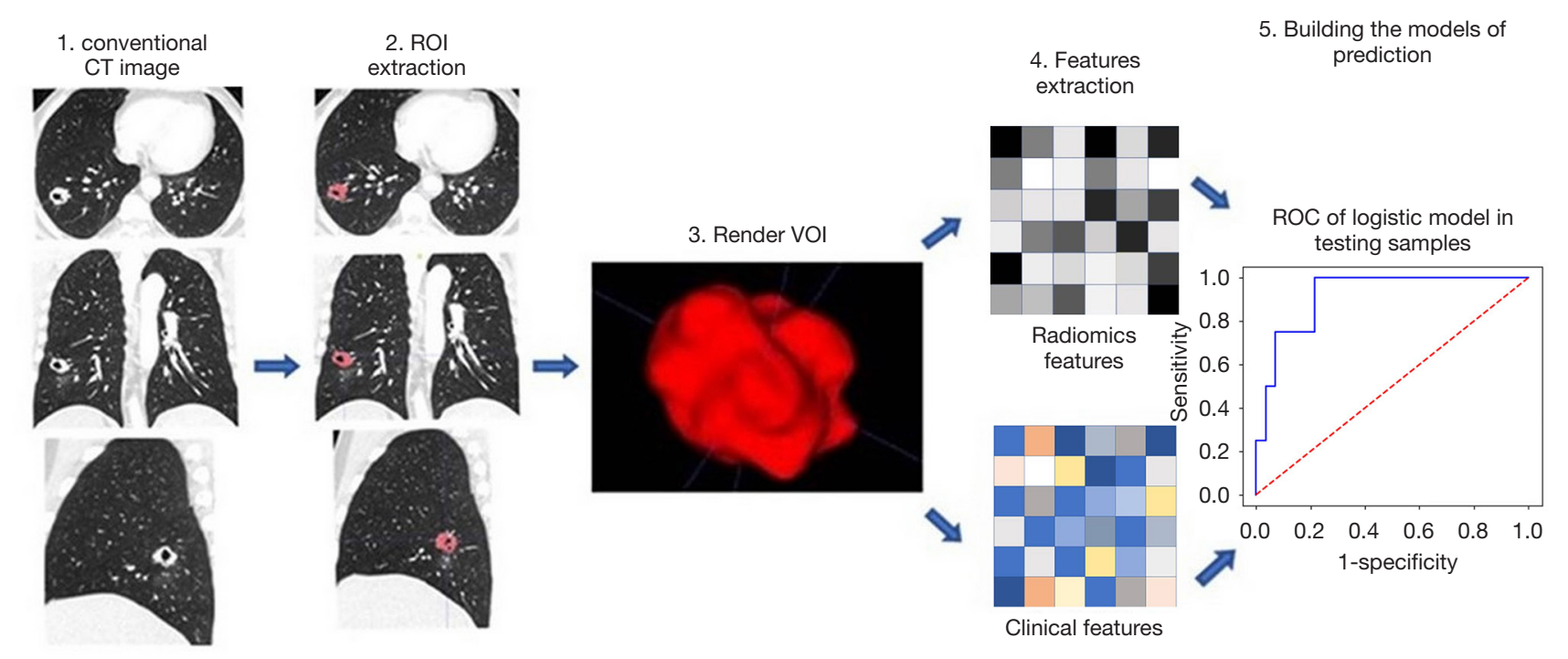

Figure 1 The flowchart shows the prediction process of radiomics. The first step is to obtain high-quality conventional CT images. The second step is to outline the lesion area from the high-quality images. The third step is to segment the region of interest (ROI) which is eventually rendered in three dimensions $(3 D)$ with specific software. The fourth step is to extract the quantitative features from these rendered volumes. The fifth step is to place the radiomic features in a database along with other data, such as clinical data. These data will been integrated, statistically analyzed, and finally mined into the optimal prediction model.

Table 1 Examples of Semantic and Agnostic Features of Radiomics (29)

\begin{tabular}{ll}
\hline Semantic & Agnostic \\
\hline Size & Histogram (skewness, kurtosis) \\
Shape & Haralick textures \\
Location & Laws textures \\
Vascularity & Wavelets \\
Spiculation & Laplacian transforms \\
Necrosis & Minkowski functionals \\
Attachments or lepidics & Fractal dimensions \\
\hline
\end{tabular}

mutation, EGFR mutation has the highest mutation rate in lung adenocarcinoma and is common in Asian patients, female patients, and nonsmokers (25,30-32). Globally, EGFR is considered the most effective predictive biomarker for treatment outcomes with first-line epidermal growth factor receptor tyrosine kinase inhibitors (EGFR-TKIs). The prognosis of lung adenocarcinoma patients with EGFR mutations is better than that of those without EGFR mutations (10,33). The common EGFR mutation subtypes often occur within the EGFR exons 18 to 21, among which deletion in exon 19 and substitution of leucine for arginine (L858R) in exon 21 account for approximately $90 \%$ of EGFR oncogenic mutation subtypes (34), both of which are sensitive to EGFR-TKI treatment (35-38). In the treatment, exon 19 deletion was associated with better outcomes than exon 21 L858R substitution $(35,36)$.

In lung adenocarcinoma, conventional CT imaging features ground-glass opacity (GGO), and a small tumour size indicate better survival (39-45). Most have shown that GGOs and smaller tumour volumes are the most prominent imaging features of lung adenocarcinoma with EGFR mutations than wild-type EGFR mutations (25,46-55). In lung adenocarcinoma with different EGFR mutation substyles, lesions with exon 19 deletion and exon $21 \mathrm{~L} 858 \mathrm{R}$ substitution are related to a higher GGO proportion than other mutation substyles, and the viewpoints that which of the two has higher GGO proportion are inconsistent in different studies (47,50,56-59). In our index, the Area under curve (AUC) values of conventional CT images for predicting EGFR mutation status were mostly lower than $0.8(46,51,59)$. Moreover, studies have showed that conventional CT imaging features of lung adenocarcinoma combined with clinical variables could better classify EGFR mutation status than clinical variables alone $(25,53,59)$. In Liu et al.'s study, the use of clinical variables combined with conventional 
$\mathrm{CT}$ imaging features (AUC $=0.778$ ) was superior to the use of clinical variables alone (AUC $=0.690)(25)$. In Han et al.'s study, AUC values of 0.647 and 0.712 for clinicalonly or combined CT imaging features, respectively, for the prediction of EGFR mutations, led to a similar conclusion and revealed a significant difference between them $(\mathrm{P}=0.0344)(53)$. However, there are still some studies showing no significant correlation between GGO status and EGFR mutations $(60,61)$. The reason the above results are different may be that the reading of conventional CT imaging features is easily affected by subjective factors, different methods of analysing GGO, a small sample size, and different grouping methods among studies.

CT image-based radiomic features can sensitively distinguish between EGFR-positive and EGFR-negative mutations in lung adenocarcinomas (62-71). Moreover, CT image-based radiomics has the capacity to distinguish EGFR subtype mutation exon 19 deletion and exon 21 L858R substitution $(64,68)$. In some studies, the predictive performance of CT image-based radiomics in the identification of EGFR mutations in lung adenocarcinoma was better than that for EGFR subtype mutations $(64,72)$, which may be due to the inclusion of clinical variables in the EGFR mutation groups. In another study, after deep learning of CT image-based radiomic features, the prediction model recognized EGFR mutation status with AUCs of 0.910 and 0.841 for the internal and external test cohorts, respectively (73), with outstanding performance. In addition, most studies show that the combination of CT image-based radiomic features and clinical variables has a better prediction effect than either approach alone (62,64-70,74,75), and moreover, CT image-based radiomic features combined with clinical and conventional CT features or pathological types can better identify EGFR mutations $(63,67-70,76)$. In most studies, clinical features generally include mean age, sex and smoking history. $\mathrm{Lu}$ et al. (67) combined CT image-based radiomic features with clinicopathological features to predict EGFR, with an AUC of 0.894 for the test cohort, which had the best distinguishing ability compared with the combined clinicopathological and conventional CT imaging features (AUC for the test dataset $=0.768$ ) and CT image-based radiomic features (AUC for the test dataset $=0.837$ ) alone. To date, their study had the best prediction result to predict EGFR mutation status in lung adenocarcinoma with a radiomic model ( $\mathrm{AUC}=0.90 \pm 0.02$ for the training, $0.88 \pm 0.11$ for the verification, 0.894 for the test dataset). Moreover, we note that in the prediction of EGFR mutation status and its subtypes in patients with lung adenocarcinoma, CT image-based radiomic features are better than conventional CT imaging features alone and the combined clinical and conventional CT imaging features (66-68,70). In Digumarthy et al.'s study (70), the AUC values for radiomic features and conventional CT imaging features for predicting EGFR mutations were 0.725 and 0.553 , respectively. In Zhang et al.'s study (76), the AUC values for radiomic features and the combined clinical and conventional CT imaging features for predicting EGFR mutation were 0.81 and 0.796 , respectively.

Of note, drug resistance can occur along with treatment, leading to treatment failure. Substitution of threonine 790 with methionine (T790M) is a main drug resistance mutation in non-small cell lung cancer. The T790M mutation accounts for approximately half of all resistance to EGFR inhibitors such as gefitinib and erlotinib $(77,78)$. A limited number of studies suggest that CT image-based radiomics can be used to predict EGFR T790M mutation $(79,80)$. Cucchiara et al. measured the copy number of EGFR mutations in body fluid and analysed the correlation between EGFR mutations and CT image-based radiomic features. The results showed that CT image-based radiomic signatures could detect the appearance of the T790M mutation with an AUC of 0.84 (79). In a recent study (80), one hundred and nine patients fit the inclusion criteria (including three aspects: histological diagnosis, suitability for segmentation of CT images before treatment, and clinical and radiological follow-up of EGFR-mutant patients), among whom, 21 patients were positive for EGFR mutations. Among the EGFR-mutant patients, 19 patients were evaluated for the T790M mutation. In this study, compared with those patients who did not develop the T790M mutation, patients who developed the T790M resistance mutation during TKI treatment showed statistically significant differences in 17 CT image-based radiomic features.

The prediction of EGFR mutation and its mutation subtypes in lung adenocarcinoma by conventional CT imaging features and CT image-based radiomics is a hot topic. The conventional CT imaging features are low-dimensional, and the prediction effect is limited. Radiomics provides a new quantitative analysis method for the prediction of EGFR gene mutations in lung adenocarcinoma, showing positive predictive value and the potential to become an alternative biomarker for identifying EGFR mutation, its subtypes and drug resistance gene mutations. However, it is worth noting that 
not all prediction signature models of CT image-based radiomics show particularly good performance. In some studies, the prediction performance of EGFR mutation status is not ideal with CT image-based radiomics alone $(66,69,74)$ and the AUC values of some articles are lower than $0.8(64,69,72,75)$, which may be related to the fact that the images were not preprocessed before data extraction. It is consistent that the predictive efficiency for EGFR mutation of the radiomic features combined with the clinical features has been improved in lung adenocarcinoma. Some studies have compared the predictive performance of radiomic features for EGFR mutation genes with that of conventional CT imaging features. According to these studies, the predictive performance of radiomic predictive models is better than that of conventional CT imaging features $(67,70,76)$. Even if clinical features are integrated into conventional CT features, their predictive performance is still inferior to that of the radiomic prediction model $(67,76)$.

\section{Conventional CT imaging features and CT image-based radiomic features predict ALK rearrangement genes in lung adenocarcinoma}

ALK rearrangement is another important molecular mutation (81), and it is also an important target for therapy $(82,83)$. Compared with chemotherapy, TKIs improved the prognosis of ALK-positive NSCLC patients $(84,85)$. Studies have shown that ALK rearrangement is common in young patients with lung adenocarcinoma, and lesions with ALK rearrangement are shown as solid nodules on CT images (51-53,60,86-89). The use of clinical variables combined with CT imaging features was superior to the use of clinical variables alone $(51,53,59)$ in predicting the ALK mutation status in lung adenocarcinoma. Conventional CT fimaging eatures can identify ALK-positive lung adenocarcinoma with reasonably strong accuracy (88). Moreover, studies revealed that lesions with ALK rearrangement were prone to lymph node, pleural, and pericardial metastasis $(51,52,87)$.

Some studies also indicate that CT image-based radiomic features have good performance in predicting ALK mutation $(74,90)$. The research results showed that CT image-based radiomic features combined with clinical features can better identify ALK mutations in lung adenocarcinoma $(74,91,92)$. Moreover, the standard postcontrast CT classifier had better performance in predicting ALK mutations than the pre-contrast CT classifier (92). The ALK and EGFR mutation statuses in patients with lung adenocarcinoma can be discriminated by the combined model incorporating CT image-based radiomic and clinical features or CT image-based radiomics alone $(74,90)$.

Owing to the low prevalence of ALK-positive lung tumours (93), their imaging characteristics and their relationship to molecular phenotypes are less known than those of EGFR-positive tumours. In terms of imaging methods with positive prediction of ALK rearrangement, there are relatively small studies on conventional CT imaging features and CT image-based radiomic features, but they still show good application prospects. Further studies with big data and prospective research are needed.

\section{Conventional CT imaging features and CT image- based radiomic features predict KRAS mutation in lung adenocarcinoma}

KRAS mutation is another common mutation in lung adenocarcinoma and more common in smokers and the Western population $(10,94)$. For many years, targeted drugs for KRAS gene mutations have been a research hotspot, but no effective clinical drugs have been developed, and KRAS is considered a type of mutation with no response to targeted therapy; it is associated with TKI resistance (95) and often indicates poor prognosis (96). However, it has been reported recently that sotorasib showed encouraging anticancer activity in patients with heavily pretreated advanced solid tumours harbouring the KRAS p.G12C mutation (97). On conventional CT images, solid and rounded masses are the main features $(51,52,61)$. Intrapulmonary and pleural metastases are rare in cases with KRAS mutation (52).

Studies predicting the KRAS mutation of lung adenocarcinoma by CT image-based radiomics are very limited. We found that the only study was guided by Rios Velazquez et al. (66). They conducted a multicentric study with four independent, large-scale cohorts to predict KRAS mutations by CT image-based radiomics. The results showed that CT image-based radiomic features were not effective in predicting KRAS mutations, and the AUC was 0.63. In this experiment, the combination of CT imagebased radiomic and clinical features (AUC $=0.69)$ to predict KRAS mutation status had higher predictive power than CT image-based radiomics (AUC $=0.63$ ) but lower predictive power than clinical features (AUC $=0.75$ ), which is different from the situation of EGFR and ALK mutation status. It is worth mentioning that the combination of clinical features and radiomic features effectively distinguished EGFR and 
KRAS mutations, with an AUC of 0.86 (66).

There are only a few studies on the prediction of KRAS mutation by CT image-based radiomics, which may be related to the lack of corresponding targeted drugs. However, existing studies show that conventional CT imaging and CT image-based radiomic features still have value in predicting KRAS mutations in lung adenocarcinoma. Conventional CT imaging features of the above three relatively common gene mutations in lung adenocarcinoma are summarized in Table 2. Predicting the above three relatively common gene mutations with CT image-based radiomic features are summarized in Table 3.

\section{Conventional CT imaging features and CT image- based radiomic features predict other rare mutations in adenocarcinoma}

There are also some rare types of mutations in lung adenocarcinoma, such as RET rearrangement and ROS1, BRAF, and HER2 mutations. Targeted therapy aimed at these rare mutations is still being studied. In terms of conventional CT imaging features, these rare mutations are characterized by solid nodules $(89,98-102)$, and each mutation has its own detailed CT characteristics in lung adenocarcinoma. ROS1-rearranged adenocarcinoma appeared as solid tumours and was associated with young age (89), pericardial metastases, and advanced nodal metastases (102). Masses with RET rearrangement have a rounded or lobulated margin and are less likely to have lymph node metastasis and commonly have pleural, lung, bone, and brain metastases $(98,99)$. BRAF mutation are often located in the peripheral lung field with burrs (100). In the peripheral lung field, pleural traction, and lymph node metastasis are common in lung adenocarcinoma of HER2 mutation, which indicates that this kind of mutation type has higher invasive potential (101). We have not found studies of CT image-based radiomic features to predict these rare mutations in lung adenocarcinoma.

\section{Shortcomings and challenges in radiomics}

In the past five years, there have been more studies predicting mutated genes with CT image-based radiomics than with conventional CT images. The high-dimensional CT image-based radiomic features produced superior identifying performance compared with that of lowdimensional conventional CT images for distinguishing the gene mutation status in lung adenocarcinoma (76).
However, the predicted results of CT image-based radiomics are partly not satisfactory $(74,75)$ and are even worse than the predicted results of clinical features alone $(66,70)$. The reasons for the instability of CT image-based radiomic performance come from many aspects of the whole process of radiomics which are also the shortcomings and challenges in radiomics. (I) Image acquisition and reconstruction protocols lack standardization. The differences of parameters of acquisition and image reconstruction algorithm can introduce changes that are not due to underlying biological effects when images are analysed numerically, where the heterogeneity of radiomic study design mainly lies in studies. To achieve reproducibility, the same or similar scanning parameters, including layer thickness, tube voltage and tube current, are needed, and the reconstruction algorithm should be standardized and generalized. However, there is still no unified CT scanning protocol or image reconstruction standard. We need further study to establish image acquisition and reconstruction protocol standardization. (II) There is no unified method for the accurate segmentation of regions of interest (ROIs). Segmenting the nodule from the adjacent region accurately is the most critical, challenging, and contentious component of radiomics, so an accurate and repeatable nodule segmentation method is needed. Some scholars believe that the combination of computeraided edge detection and manual segmentation can achieve the best results, but there is no consensus on which method is better $(29,103)$. (III) In application, the specific algorithm of radiomics cannot be explained. The feature extraction part is not transparent. How to make clinicians trust this tool is another problem. (IV) Most of the studies based on radiomics are retrospective studies with small sample sizes, which is another reason for the heterogeneity of study design in studies. There is a lack of multiple-site, largecohort, prospective studies. Ideally, current studies should be combined to provide a meta-analysis, which is what our research team intends to do next.

\section{Summary and prospect}

In general, conventional CT images provide some information for predicting lung adenocarcinoma gene mutations, however, some features overlap and lack specificity, and it is subjective and lacks objective basis. CT image-based radiomics provides a potential noninvasive method for the prediction of different gene mutations in lung adenocarcinoma when surgery and 
Table 2 Conventional CT features of different mutated genes in lung adenocarcinoma

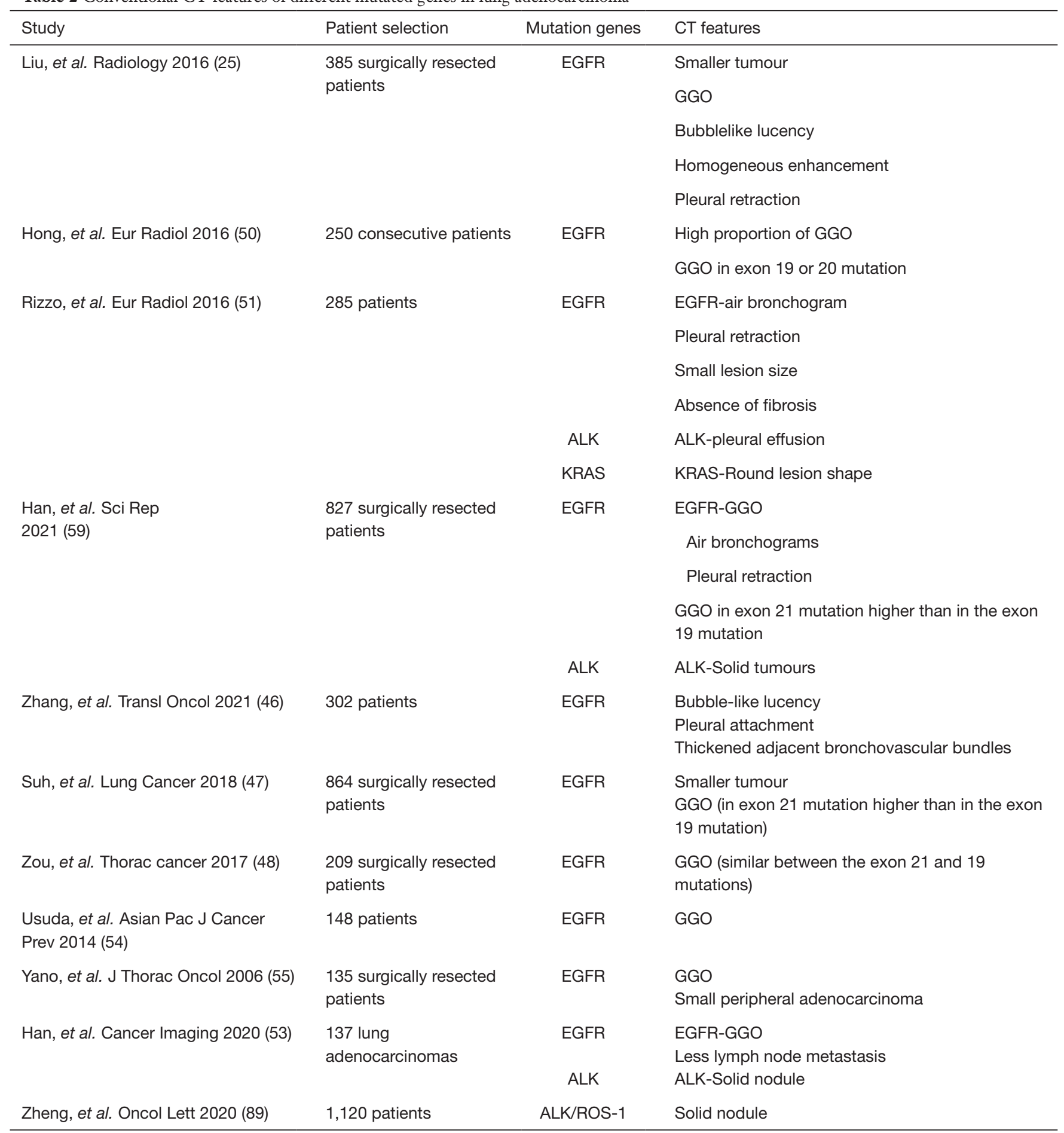

Table 2 (continued) 
Table 2 (continued)

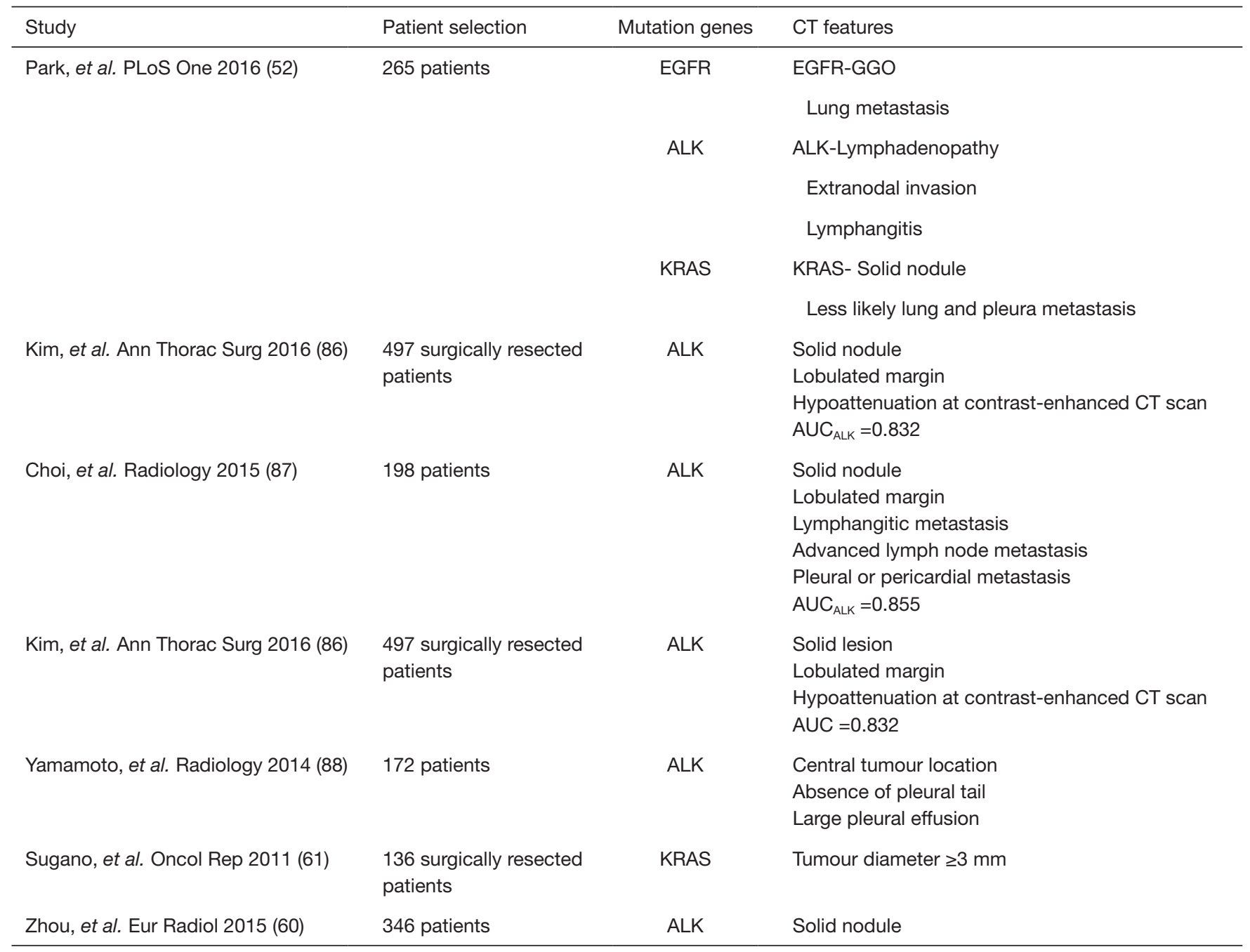

CT, computed tomography; EGFR, epidermal growth factor receptor; GGO, ground glass opacity; ALK, anaplastic Iymphoma kinase; KRAS, Kirsten rat sarcoma viral oncogene; AUC, Area Under the Curve.

biopsy are not available and radiomics models comparing clinical features could help to make treatment decisions. Artificial intelligence is an irreversible trend of medical development. As a primary part of artificial intelligence, in recent years, radiomics studies have been repeated without new breakthroughs. The author believes that the precise automatic recognition of artificial intelligence will replace the manual sketch of radiomics, the process that the sketched region of interest submitted to radiomics company for processing will be replaced by one click analysis software, and deep learning, such as the neural network, will replace simple machine learning in the classification of gene mutation in lung adenocarcinoma. Research on the application of conventional CT imaging features and CT image-based radiomic features for predicting the gene mutation status of lung adenocarcinoma is still in a preliminary stage. Although there is some hope that radiomics could be applied in clinical practice, more work 


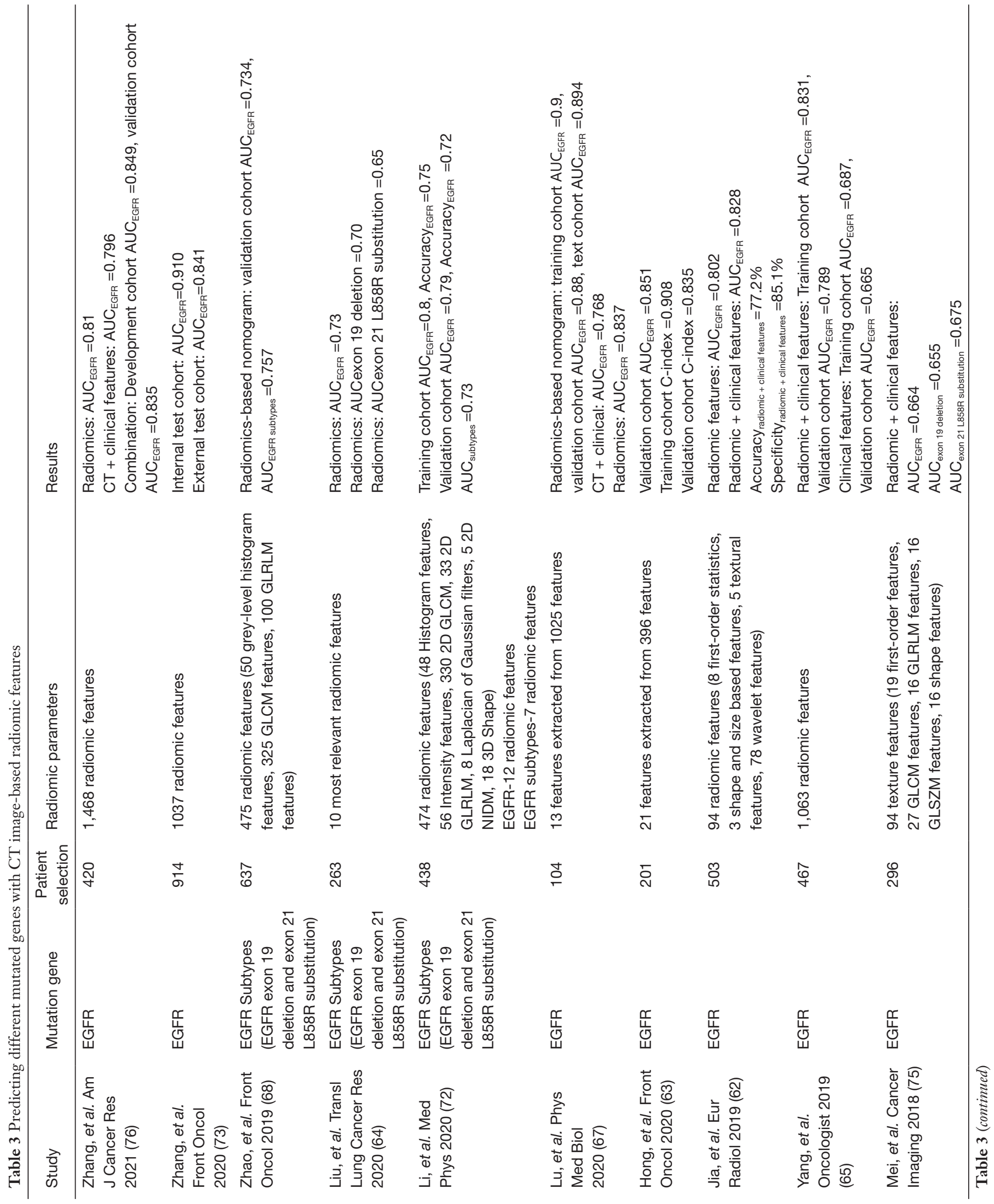




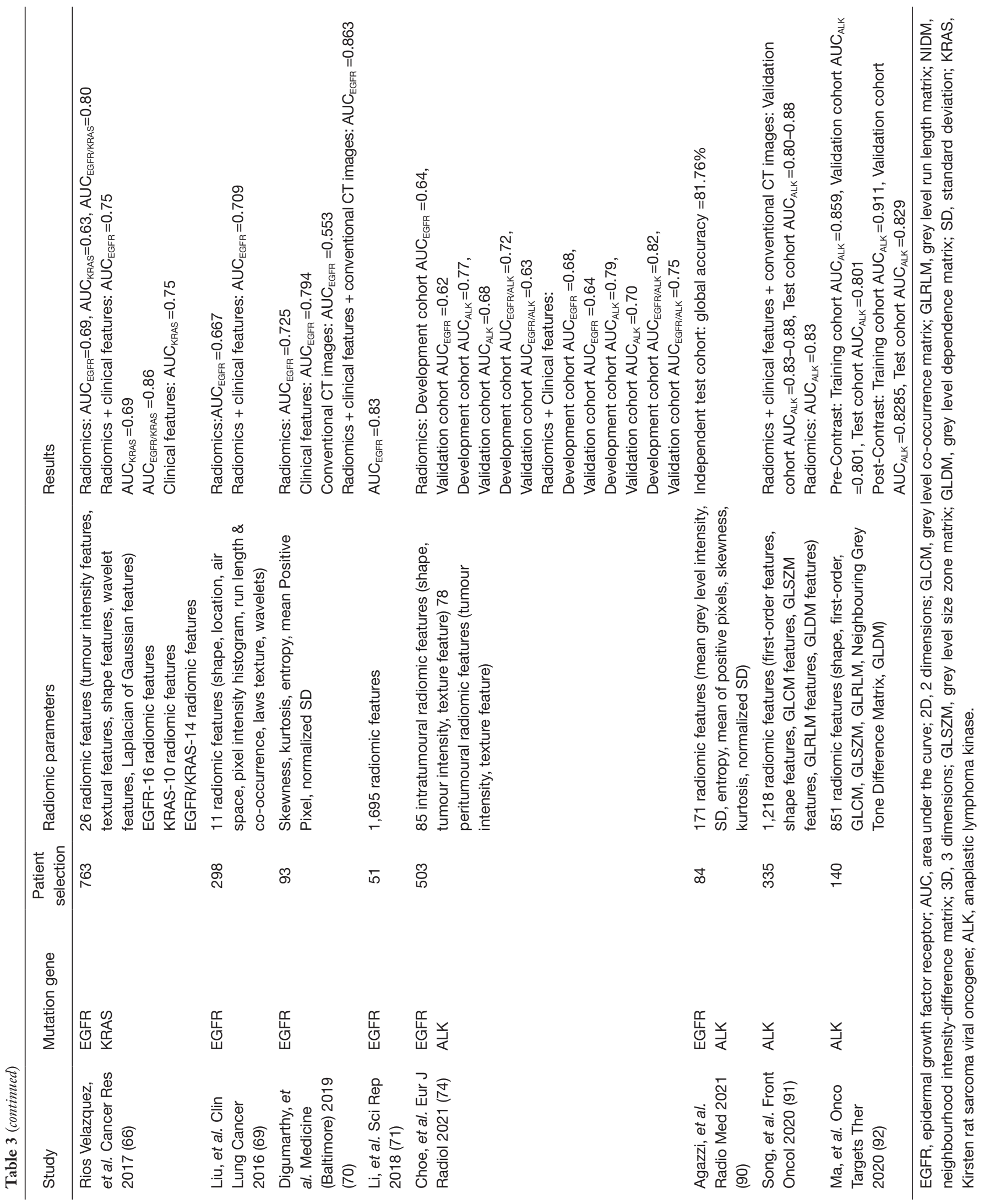


needs to be done.

\section{Acknowledgments}

Funding: None.

\section{Footnote}

Reporting Checklist: The authors have completed the Narrative Review reporting checklist. Available at https:// dx.doi.org/10.21037/tcr-21-1037

Peer Review File: Available at https://dx.doi.org/10.21037/ tcr-21-1037

Conflicts of Interest: Both authors have completed the ICMJE uniform disclosure form (available at https://dx.doi. org/10.21037/tcr-21-1037). The authors have no conflicts of interest to declare.

Ethical Statement: The authors are accountable for all aspects of the work in ensuring that questions related to the accuracy or integrity of any part of the work are appropriately investigated and resolved.

Open Access Statement: This is an Open Access article distributed in accordance with the Creative Commons Attribution-NonCommercial-NoDerivs 4.0 International License (CC BY-NC-ND 4.0), which permits the noncommercial replication and distribution of the article with the strict proviso that no changes or edits are made and the original work is properly cited (including links to both the formal publication through the relevant DOI and the license). See: https://creativecommons.org/licenses/by-nc-nd/4.0/.

\section{References}

1. Safiri S, Sohrabi MR, Carson-Chahhoud K, et al. Burden of Tracheal, Bronchus, and Lung Cancer and Its Attributable Risk Factors in 204 Countries and Territories, 1990 to 2019. J Thorac Oncol 2021;16:945-59.

2. Siegel RL, Miller KD, Fuchs HE, et al. Cancer Statistics, 2021. CA Cancer J Clin 2021;71:7-33.

3. Yu YW, Wang CP, Han YF, et al. Meta-analysis on related risk factors regarding lung cancer in non-smoking Chinese women. Zhonghua Liu Xing Bing Xue Za Zhi 2016;37:268-72.

4. Lin KF, Wu HF, Huang WC, et al. Propensity score analysis of lung cancer risk in a population with high prevalence of non-smoking related lung cancer. BMC Pulm Med 2017;17:120.

5. Detterbeck FC, Nicholson AG, Franklin WA, et al. The IASLC Lung Cancer Staging Project: Summary of Proposals for Revisions of the Classification of Lung Cancers with Multiple Pulmonary Sites of Involvement in the Forthcoming Eighth Edition of the TNM Classification. J Thorac Oncol 2016;11:639-50.

6. Subramanian J, Govindan R. Lung cancer in never smokers: a review. J Clin Oncol 2007;25:561-70.

7. Wu FZ, Chen PA, Wu CC, et al. Semiquantative Visual Assessment of Sub-solid Pulmonary Nodules $\leq 3 \mathrm{~cm}$ in Differentiation of Lung Adenocarcinoma Spectrum. Sci Rep 2017;7:15790.

8. Wu FZ, Huang YL, Wu CC, et al. Assessment of Selection Criteria for Low-Dose Lung Screening CT Among Asian Ethnic Groups in Taiwan: From Mass Screening to Specific Risk-Based Screening for Non-Smoker Lung Cancer. Clin Lung Cancer 2016;17:e45-56.

9. Rosell R, Carcereny E, Gervais R, et al. Erlotinib versus standard chemotherapy as first-line treatment for European patients with advanced EGFR mutation-positive non-small-cell lung cancer (EURTAC): a multicentre, open-label, randomised phase 3 trial. Lancet Oncol 2012;13:239-46.

10. Guan JL, Zhong WZ, An SJ, et al. KRAS mutation in patients with lung cancer: a predictor for poor prognosis but not for EGFR-TKIs or chemotherapy. Ann Surg Oncol 2013;20:1381-8.

11. Meng D, Yuan M, Li X, et al. Prognostic value of K-RAS mutations in patients with non-small cell lung cancer: a systematic review with meta-analysis. Lung Cancer 2013;81:1-10.

12. Woo T, Okudela K, Yazawa T, et al. Prognostic value of KRAS mutations and Ki-67 expression in stage I lung adenocarcinomas. Lung Cancer 2009;65:355-62.

13. Li P, Gao Q, Jiang X, et al. Comparison of Clinicopathological Features and Prognosis between ALK Rearrangements and EGFR Mutations in Surgically Resected Early-stage Lung Adenocarcinoma. J Cancer 2019;10:61-71.

14. Tao H, Cai Y, Shi L, et al. Analysis of clinical characteristics and prognosis of patients with anaplastic lymphoma kinase-positive and surgically resected lung adenocarcinoma. Thorac Cancer 2017;8:8-15.

15. Liu Y, Ye X, Yu Y, et al. Prognostic significance of anaplastic lymphoma kinase rearrangement in patients 
with completely resected lung adenocarcinoma. J Thorac Dis 2019;11:4258-70.

16. Dong Y, Li Y, Jin B, et al. Pathologic subtype-defined prognosis is dependent on both tumor stage and status of oncogenic driver mutations in lung adenocarcinoma. Oncotarget 2017;8:82244-55.

17. Hsiao SH, Lin HC, Chou Y'T, et al. Impact of epidermal growth factor receptor mutations on intracranial treatment response and survival after brain metastases in lung adenocarcinoma patients. Lung Cancer 2013;81:455-61.

18. Kosaka T, Yatabe Y, Onozato R, et al. Prognostic implication of EGFR, KRAS, and TP53 gene mutations in a large cohort of Japanese patients with surgically treated lung adenocarcinoma. J Thorac Oncol 2009;4:22-9.

19. Robertson EG, Baxter G. Tumour seeding following percutaneous needle biopsy: the real story! Clin Radiol 2011;66:1007-14.

20. Shyamala K, Girish HC, Murgod S. Risk of tumor cell seeding through biopsy and aspiration cytology. J Int Soc Prev Community Dent 2014;4:5-11.

21. Pascoe HM, Knipe HC, Pascoe D, et al. The many faces of lung adenocarcinoma: A pictorial essay. J Med Imaging Radiat Oncol 2018;62:654-61.

22. Hansell DM, Bankier AA, MacMahon H, et al. Fleischner Society: glossary of terms for thoracic imaging. Radiology 2008;246:697-722.

23. Gaikwad A, Gupta A, Hare S, et al. Primary adenocarcinoma of lung: a pictorial review of recent updates. Eur J Radiol 2012;81:4146-55.

24. Yoshida T, Harada T, Fuke S, et al. Lung adenocarcinoma presenting with enlarged and multiloculated cystic lesions over 2 years. Respir Care 2004;49:1522-4.

25. Liu Y, Kim J, Qu F, et al. CT Features Associated with Epidermal Growth Factor Receptor Mutation Status in Patients with Lung Adenocarcinoma. Radiology 2016;280:271-80.

26. Balagurunathan Y, Gu Y, Wang H, et al. Reproducibility and Prognosis of Quantitative Features Extracted from CT Images. Transl Oncol 2014;7:72-87.

27. Zhou M, Leung A, Echegaray S, et al. Non-Small Cell Lung Cancer Radiogenomics Map Identifies Relationships between Molecular and Imaging Phenotypes with Prognostic Implications. Radiology 2018;286:307-15.

28. Aerts HJ, Velazquez ER, Leijenaar RT, et al. Decoding tumour phenotype by noninvasive imaging using a quantitative radiomics approach. Nat Commun 2014;5:4006.

29. Gillies RJ, Kinahan PE, Hricak H. Radiomics: Images
Are More than Pictures, They Are Data. Radiology 2016;278:563-77.

30. Yatabe Y, Kerr KM, Utomo A, et al. EGFR mutation testing practices within the Asia Pacific region: results of a multicenter diagnostic survey. J Thorac Oncol 2015;10:438-45.

31. Shigematsu H, Lin L, Takahashi T, et al. Clinical and biological features associated with epidermal growth factor receptor gene mutations in lung cancers. J Natl Cancer Inst 2005;97:339-46.

32. da Cunha Santos G, Shepherd FA, Tsao MS. EGFR mutations and lung cancer. Annu Rev Pathol 2011;6:49-69.

33. Tan CS, Gilligan D, Pacey S. Treatment approaches for EGFR-inhibitor-resistant patients with non-small-cell lung cancer. Lancet Oncol 2015;16:e447-59.

34. Zhu JQ, Zhong WZ, Zhang GC, et al. Better survival with EGFR exon 19 than exon 21 mutations in gefitinibtreated non-small cell lung cancer patients is due to differential inhibition of downstream signals. Cancer Lett 2008;265:307-17.

35. Zhou J, Ben S. Comparison of therapeutic effects of EGFR-tyrosine kinase inhibitors on 19Del and L858R mutations in advanced lung adenocarcinoma and effect on cellular immune function. Thorac Cancer 2018;9:228-33.

36. He Q, Xin P, Zhang M, et al. The impact of epidermal growth factor receptor mutations on the prognosis of resected non-small cell lung cancer: a meta-analysis of literatures. Transl Lung Cancer Res 2019;8:124-34.

37. Rosell R, Moran T, Queralt C, et al. Screening for epidermal growth factor receptor mutations in lung cancer. N Engl J Med 2009;361:958-67.

38. Mok TS, Wu YL, Thongprasert S, et al. Gefitinib or carboplatin-paclitaxel in pulmonary adenocarcinoma. N Engl J Med 2009;361:947-57.

39. Mao R, She Y, Zhu E, et al. A Proposal for Restaging of Invasive Lung Adenocarcinoma Manifesting as Pure Ground Glass Opacity. Ann Thorac Surg 2019;107:1523-31.

40. Wilshire CL, Louie BE, Manning KA, et al. Radiologic Evaluation of Small Lepidic Adenocarcinomas to Guide Decision Making in Surgical Resection. Ann Thorac Surg 2015;100:979-88.

41. Takahashi M, Shigematsu Y, Ohta M, et al. Tumor invasiveness as defined by the newly proposed IASLC/ ATS/ERS classification has prognostic significance for pathologic stage IA lung adenocarcinoma and can be predicted by radiologic parameters. J Thorac Cardiovasc Surg 2014;147:54-9. 
42. Tsubochi H, Sakaguchi H, Yamasaki N, et al. Clinicopathologic analysis of small-sized non-small cell lung cancer. Kyobu Geka 2012;65:4-10.

43. Asamura H, Suzuki K, Watanabe S, et al. A clinicopathological study of resected subcentimeter lung cancers: a favorable prognosis for ground glass opacity lesions. Ann Thorac Surg 2003;76:1016-22.

44. Cibas ES, Melamed MR, Zaman MB, et al. The effect of tumor size and tumor cell DNA content on the survival of patients with stage I adenocarcinoma of the lung. Cancer 1989;63:1552-6.

45. Lee SY, Jeon JH, Jung W, et al. Predictive Factors for Lymph Node Metastasis in Clinical Stage I Part-Solid Lung Adenocarcinoma. Ann Thorac Surg 2021;111:456-62.

46. Zhang G, Zhang J, Cao Y, et al. Nomogram based on preoperative CT imaging predicts the EGFR mutation status in lung adenocarcinoma. Transl Oncol 2021;14:100954.

47. Suh YJ, Lee HJ, Kim YJ, et al. Computed tomography characteristics of lung adenocarcinomas with epidermal growth factor receptor mutation: A propensity score matching study. Lung Cancer 2018;123:52-9.

48. Zou J, Lv T, Zhu S, et al. Computed tomography and clinical features associated with epidermal growth factor receptor mutation status in stage I/II lung adenocarcinoma. Thorac Cancer 2017;8:260-70.

49. Hasegawa M, Sakai F, Ishikawa R, et al. CT Features of Epidermal Growth Factor Receptor-Mutated Adenocarcinoma of the Lung: Comparison with Nonmutated Adenocarcinoma. J Thorac Oncol 2016;11:819-26.

50. Hong SJ, Kim TJ, Choi YW, et al. Radiogenomic correlation in lung adenocarcinoma with epidermal growth factor receptor mutations: Imaging features and histological subtypes. Eur Radiol 2016;26:3660-8.

51. Rizzo S, Petrella F, Buscarino V, et al. CT Radiogenomic Characterization of EGFR, K-RAS, and ALK Mutations in Non-Small Cell Lung Cancer. Eur Radiol 2016;26:32-42.

52. Park J, Kobayashi Y, Urayama KY, et al. Imaging Characteristics of Driver Mutations in EGFR, KRAS, and ALK among Treatment-Naïve Patients with Advanced Lung Adenocarcinoma. PLoS One 2016;11:e0161081.

53. Han X, Fan J, Gu J, et al. CT features associated with EGFR mutations and ALK positivity in patients with multiple primary lung adenocarcinomas. Cancer Imaging 2020;20:51.

54. Usuda K, Sagawa M, Motono N, et al. Relationships between EGFR mutation status of lung cancer and preoperative factors - are they predictive? Asian Pac J Cancer Prev 2014;15:657-62.

55. Yano M, Sasaki H, Kobayashi Y, et al. Epidermal growth factor receptor gene mutation and computed tomographic findings in peripheral pulmonary adenocarcinoma. J Thorac Oncol 2006;1:413-6.

56. Lee HJ, Kim YT, Kang CH, et al. Epidermal growth factor receptor mutation in lung adenocarcinomas: relationship with CT characteristics and histologic subtypes. Radiology 2013;268:254-64.

57. Yoshida Y, Kokubu A, Suzuki K, et al. Molecular markers and changes of computed tomography appearance in lung adenocarcinoma with ground-glass opacity. Jpn J Clin Oncol 2007;37:907-12.

58. Yang Y, Yang Y, Zhou X, et al. EGFR L858R mutation is associated with lung adenocarcinoma patients with dominant ground-glass opacity. Lung Cancer 2015;87:272-7.

59. Han X, Fan J, Li Y, et al. Value of CT features for predicting EGFR mutations and ALK positivity in patients with lung adenocarcinoma. Sci Rep 2021;11:5679.

60. Zhou JY, Zheng J, Yu ZF, et al. Comparative analysis of clinicoradiologic characteristics of lung adenocarcinomas with ALK rearrangements or EGFR mutations. Eur Radiol 2015;25:1257-66.

61. Sugano M, Shimizu K, Nakano T, et al. Correlation between computed tomography findings and epidermal growth factor receptor and KRAS gene mutations in patients with pulmonary adenocarcinoma. Oncol Rep 2011;26:1205-11.

62. Jia TY, Xiong JF, Li XY, et al. Identifying EGFR mutations in lung adenocarcinoma by noninvasive imaging using radiomics features and random forest modeling. Eur Radiol 2019;29:4742-50.

63. Hong D, Xu K, Zhang L, et al. Radiomics Signature as a Predictive Factor for EGFR Mutations in Advanced Lung Adenocarcinoma. Front Oncol 2020;10:28.

64. Liu $\mathrm{G}, \mathrm{Xu} Z$, Ge Y, et al. 3D radiomics predicts EGFR mutation, exon-19 deletion and exon-21 L858R mutation in lung adenocarcinoma. Transl Lung Cancer Res 2020;9:1212-24.

65. Yang X, Dong X, Wang J, et al. Computed TomographyBased Radiomics Signature: A Potential Indicator of Epidermal Growth Factor Receptor Mutation in Pulmonary Adenocarcinoma Appearing as a Subsolid Nodule. Oncologist 2019;24:e1156-64.

66. Rios Velazquez E, Parmar C, Liu Y, et al. Somatic Mutations Drive Distinct Imaging Phenotypes in Lung 
Cancer. Cancer Res 2017;77:3922-30.

67. Lu X, Li M, Zhang H, et al. A novel radiomic nomogram for predicting epidermal growth factor receptor mutation in peripheral lung adenocarcinoma. Phys Med Biol 2020;65:055012.

68. Zhao W, Wu Y, Xu Y, et al. The Potential of Radiomics Nomogram in Non-invasively Prediction of Epidermal Growth Factor Receptor Mutation Status and Subtypes in Lung Adenocarcinoma. Front Oncol 2020;9:1485.

69. Liu Y, Kim J, Balagurunathan Y, et al. Radiomic Features Are Associated With EGFR Mutation Status in Lung Adenocarcinomas. Clin Lung Cancer 2016;17:441-448.e6.

70. Digumarthy SR, Padole AM, Gullo RL, et al. Can CT radiomic analysis in NSCLC predict histology and EGFR mutation status? Medicine (Baltimore) 2019;98:e13963.

71. Li Y, Lu L, Xiao M, et al. CT Slice Thickness and Convolution Kernel Affect Performance of a Radiomic Model for Predicting EGFR Status in Non-Small Cell Lung Cancer: A Preliminary Study. Sci Rep 2018;8:17913.

72. Li S, Luo T, Ding C, et al. Detailed identification of epidermal growth factor receptor mutations in lung adenocarcinoma: Combining radiomics with machine learning. Med Phys 2020;47:3458-66.

73. Zhang B, Qi S, Pan X, et al. Deep CNN Model Using CT Radiomics Feature Mapping Recognizes EGFR Gene Mutation Status of Lung Adenocarcinoma. Front Oncol 2021;10:598721.

74. Choe J, Lee SM, Kim W, et al. CT radiomics-based prediction of anaplastic lymphoma kinase and epidermal growth factor receptor mutations in lung adenocarcinoma. Eur J Radiol 2021;139:109710.

75. Mei D, Luo Y, Wang Y, et al. CT texture analysis of lung adenocarcinoma: can Radiomic features be surrogate biomarkers for EGFR mutation statuses. Cancer Imaging 2018;18:52.

76. Zhang G, Cao Y, Zhang J, et al. Predicting EGFR mutation status in lung adenocarcinoma: development and validation of a computed tomography-based radiomics signature. Am J Cancer Res 2021;11:546-60.

77. Kosaka T, Yatabe Y, Endoh H, et al. Analysis of epidermal growth factor receptor gene mutation in patients with nonsmall cell lung cancer and acquired resistance to gefitinib. Clin Cancer Res 2006;12:5764-9.

78. Balak MN, Gong Y, Riely GJ, et al. Novel D761Y and common secondary T790M mutations in epidermal growth factor receptor-mutant lung adenocarcinomas with acquired resistance to kinase inhibitors. Clin Cancer Res 2006;12:6494-501
79. Cucchiara F, Del Re M, Valleggi S, et al. Integrating Liquid Biopsy and Radiomics to Monitor Clonal Heterogeneity of EGFR-Positive Non-Small Cell Lung Cancer. Front Oncol 2020;10:593831.

80. Rossi G, Barabino E, Fedeli A, et al. Radiomic Detection of EGFR Mutations in NSCLC. Cancer Res 2021;81:724-31.

81. Zhao R, Zhang J, Han Y, et al. Clinicopathological Features of ALK Expression in 9889 Cases of Nonsmall-Cell Lung Cancer and Genomic Rearrangements Identified by Capture-Based Next-Generation Sequencing: A Chinese Retrospective Analysis. Mol Diagn Ther 2019;23:395-405.

82. Minguet J, Smith KH, Bramlage P. Targeted therapies for treatment of non-small cell lung cancer--Recent advances and future perspectives. Int J Cancer 2016;138:2549-61.

83. Thai AA, Solomon BJ. Treatment of ALK-positive nonsmall cell lung cancer: recent advances. Curr Opin Oncol 2018;30:84-91.

84. Zhang YC, Zhou Q, Wu YL. Efficacy of crizotinib in first-line treatment of adults with ALK-positive advanced NSCLC. Expert Opin Pharmacother 2016;17:1693-701.

85. Wu YL, Lu S, Lu Y, et al. Results of PROFILE 1029, a Phase III Comparison of First-Line Crizotinib versus Chemotherapy in East Asian Patients with ALK-Positive Advanced Non-Small Cell Lung Cancer. J Thorac Oncol 2018;13:1539-48.

86. Kim TJ, Lee CT, Jheon SH, et al. Radiologic Characteristics of Surgically Resected Non-Small Cell Lung Cancer With ALK Rearrangement or EGFR Mutations. Ann Thorac Surg 2016;101:473-80.

87. Choi CM, Kim MY, Hwang HJ, et al. Advanced adenocarcinoma of the lung: comparison of CT characteristics of patients with anaplastic lymphoma kinase gene rearrangement and those with epidermal growth factor receptor mutation. Radiology 2015;275:272-9.

88. Yamamoto S, Korn RL, Oklu R, et al. ALK molecular phenotype in non-small cell lung cancer: CT radiogenomic characterization. Radiology 2014;272:568-76.

89. Zheng J, Zhou J, Liu J, et al. Quantitative volumetric assessment of the solid portion percentage on CT images to predict ROS1/ALK rearrangements in lung adenocarcinomas. Oncol Lett 2020;20:2987-96.

90. Agazzi GM, Ravanelli M, Roca E, et al. CT texture analysis for prediction of EGFR mutational status and ALK rearrangement in patients with non-small cell lung cancer. Radiol Med 2021;126:786-94.

91. Song L, Zhu Z, Mao L, et al. Clinical, Conventional CT 
and Radiomic Feature-Based Machine Learning Models for Predicting ALK Rearrangement Status in Lung Adenocarcinoma Patients. Front Oncol 2020;10:369.

92. Ma DN, Gao XY, Dan YB, et al. Evaluating Solid Lung Adenocarcinoma Anaplastic Lymphoma Kinase Gene Rearrangement Using Noninvasive Radiomics Biomarkers. Onco Targets Ther 2020;13:6927-35.

93. Pan Y, Zhang Y, Li Y, et al. ALK, ROS1 and RET fusions in 1139 lung adenocarcinomas: a comprehensive study of common and fusion pattern-specific clinicopathologic, histologic and cytologic features. Lung Cancer 2014;84:121-6.

94. Eberhard DA, Johnson BE, Amler LC, et al. Mutations in the epidermal growth factor receptor and in KRAS are predictive and prognostic indicators in patients with non-small-cell lung cancer treated with chemotherapy alone and in combination with erlotinib. J Clin Oncol 2005;23:5900-9.

95. Massarelli E, Varella-Garcia M, Tang X, et al. KRAS mutation is an important predictor of resistance to therapy with epidermal growth factor receptor tyrosine kinase inhibitors in non-small-cell lung cancer. Clin Cancer Res 2007;13:2890-6.

96. Lohinai Z, Klikovits T, Moldvay J, et al. KRAS-mutation incidence and prognostic value are metastatic site-specific in lung adenocarcinoma: poor prognosis in patients with KRAS mutation and bone metastasis. Sci Rep 2017;7:39721.

97. Hong DS, Fakih MG, Strickler JH, et al. KRASG12C

Cite this article as: $\mathrm{Ma} \mathrm{JW}, \mathrm{Li} \mathrm{M}$. Molecular typing of lung adenocarcinoma with computed tomography and CT imagebased radiomics: a narrative review of research progress and prospects. Transl Cancer Res 2021;10(9):4217-4231. doi: $10.21037 /$ tcr-21-1037
Inhibition with Sotorasib in Advanced Solid Tumors. N Engl J Med 2020;383:1207-17.

98. Plodkowski AJ, Drilon A, Halpenny DF, et al. From genotype to phenotype: Are there imaging characteristics associated with lung adenocarcinomas harboring RET and ROS1 rearrangements? Lung Cancer 2015;90:321-5.

99. Digumarthy SR, Mendoza DP, Lin JJ, et al. Imaging Features and Patterns of Metastasis in Non-Small Cell Lung Cancer with RET Rearrangements. Cancers (Basel) 2020;12:693.

100. Villaruz LC, Socinski MA, Abberbock S, et al. Clinicopathologic features and outcomes of patients with lung adenocarcinomas harboring BRAF mutations in the Lung Cancer Mutation Consortium. Cancer 2015;121:448-56.

101. Sawan P, Plodkowski AJ, Li AE, et al. CT features of HER2-mutant lung adenocarcinomas. Clin Imaging 2018;51:279-83.

102. Woo JH, Kim TJ, Kim TS, et al. CT features and disease spread patterns in ROS1-rearranged lung adenocarcinomas: comparison with those of EGFRmutant or ALK-rearranged lung adenocarcinomas. Sci Rep 2020;10:16251.

103. Heye T, Merkle EM, Reiner CS, et al. Reproducibility of dynamic contrast-enhanced MR imaging. Part II. Comparison of intra- and interobserver variability with manual region of interest placement versus semiautomatic lesion segmentation and histogram analysis. Radiology 2013;266:812-21. 